\title{
Neuroactive Steroids and Affective Symptoms in Women Across the Weight Spectrum
}

\author{
Laura E Dichtel ${ }^{*, 1}$, Elizabeth A Lawson', Melanie Schorr', Erinne Meenaghan², Margaret Lederfine Paskal', \\ Kamryn T Eddy ${ }^{3}$, Graziano Pinna ${ }^{4}$, Marianela Nelson ${ }^{4}$, Ann M Rasmusson ${ }^{5,6}$, Anne Klibanski' and \\ Karen K Miller
}

'Neuroendocrine Unit, Massachusetts General Hospital/Harvard Medical School, Boston, MA, USA; ${ }^{2}$ Neuroendocrine Unit, Massachusetts General Hospital, Boston, MA, USA; ${ }^{3}$ Eating Disorders Clinical and Research Program, Massachusetts General Hospital/Harvard Medical School, Boston, MA, USA; ${ }^{4}$ The Psychiatric Institute, Department of Psychiatry, University of Illinois at Chicago, Chicago, IL, USA; ${ }^{5}$ National Center for PTSD, Department of Veterans Affairs, VA Boston Healthcare System, Boston, MA, USA; ${ }^{6}$ Department of Psychiatry, Boston University School of Medicine, Boston, MA, USA

\begin{abstract}
$3 \alpha$-5 $\alpha$-Tetrahydroprogesterone, a progesterone metabolite also known as allopregnanolone, and $5 \alpha$-androstane-3 $\alpha$, I7 $\beta$-diol, a

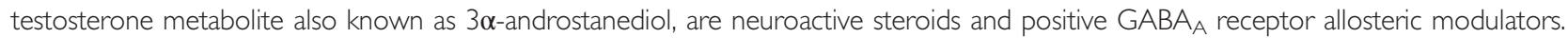
Both anorexia nervosa (AN) and obesity are complicated by affective comorbidities and hypothalamic-pituitary-gonadal dysregulation. However, it is not known whether neuroactive steroid levels are abnormal at the extremes of the weight spectrum. We hypothesized that serum allopregnanolone and $3 \alpha$-androstanediol levels would be decreased in AN compared with healthy controls ( $\mathrm{HC}$ ) and negatively associated with affective symptoms throughout the weight spectrum, independent of body mass index (BMI). Thirty-six women were I : I age-matched across three groups: AN, HC, and overweight/obese (OW/OB). AN were amenorrheic; $\mathrm{HC}$ and OW/OB were studied in the follicular phase. Fasting serum neuroactive steroids were measured by gas chromatography/mass spectrometry. Mean Hamilton depression and anxiety scores were highest in $\mathrm{AN}(p<0.000 \mathrm{I})$. Mean serum allopregnanolone was lower in $\mathrm{AN}$ and $\mathrm{OW} / \mathrm{OB}$ than $\mathrm{HC}$ (AN $95.3 \pm 56.4$ vs $\mathrm{OW} / \mathrm{OB} 73.8 \pm 31.3$ vs $\mathrm{HC} 199.5 \pm 167.8 \mathrm{pg} / \mathrm{ml}, p=0.0 \mathrm{l}$ ), despite comparable mean serum progesterone. Allopregnanolone levels, but not progesterone levels, were negatively associated with depression and anxiety symptom severity, independent of BMI. Serum 3 $\alpha$-androstanediol levels did not differ among groups and were not associated with depression or anxiety scores, despite a significant negative association between free testosterone levels and both anxiety and depression severity. In conclusion, women at both extremes of the weight spectrum have low mean serum allopregnanolone, which is associated with increased depression and anxiety severity, independent of BMI. Neuroactive steroids such as allopregnanolone may be potential therapeutic targets for depression and anxiety in traditionally treatment-resistant groups, including AN.

Neuropsychopharmacology (20I8) 43, I436-|444; doi:I0.1038/npp.2017.269; published online 6 December 2017
\end{abstract}

\section{INTRODUCTION}

Peripherally derived sex steroids, including progesterone and testosterone, can be converted by biosynthetic enzymes into neuroactive steroids that reach and act in the brain, where they may regulate affective symptoms by modulating neurotransmitter systems. The enzymes $5 \alpha$-reductase and

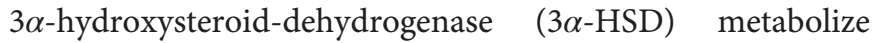
progesterone to $3 \alpha-5 \alpha$-tetrahydroprogesterone (also known as allopregnanolone) and testosterone to $5 \alpha$-androstane$3 \alpha, 17 \beta$-diol (also known as $3 \alpha$-androstanediol) in a parallel manner (Figure 1) (King, 2013; Reddy, 2010). Allopregnanolone

*Correspondence: Dr LE Dichtel, Neuroendocrine Unit, Massachusetts General Hospital, BUL457B, 55 Fruit Street, Boston, MA 02114, USA, Tel: + | 617726 3870, Fax: + I 6177265072 ,

E-mail: Idichtel@partners.org

Received 22 May 2017; revised 24 August 2017; accepted 27 October 2017; accepted article preview online I November 2017 is a potent positive allosteric modulator of GABA action at $\mathrm{GABA}_{\mathrm{A}}$ receptors increasing GABA activity with 10 times the potency of benzodiazepines (King, 2013; Majewska et al, 1986; Morrow et al, 1987). Although less potent than allopregnanolone, $3 \alpha$-androstanediol is also a positive modulator of $\mathrm{GABA}_{\mathrm{A}}$ receptor action (Reddy, 2004). Increases in $\mathrm{GABA}_{\mathrm{A}}$ receptor activity dampen neuronal excitation and result in anxiolytic, antidepressant, and sedative effects in humans (Eser et al, 2006a; Eser et al, 2006b; Rasmusson et al, 2006; Rupprecht and Holsboer, 1999a,b,; Sigel and Steinmann, 2012). However, little is known about whether such GABAergic neuroactive steroids contribute to the etiopathology of affective disorders in women at the extremes of the weight spectrum, which are enriched for both depression and anxiety symptoms.

Small studies have suggested that both cerebrospinal fluid (CSF) and serum allopregnanolone levels may be low in patients with major depressive disorder (MDD) and posttraumatic stress disorder (PTSD) as compared with healthy 


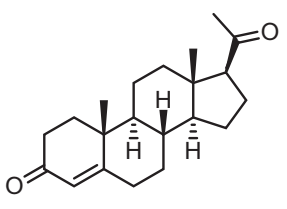

Progesterone

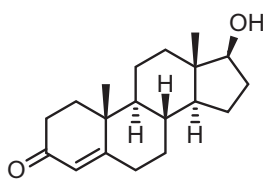

Testosterone

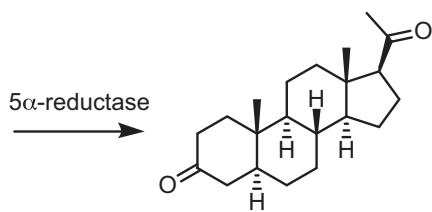

$5 \alpha$-Dihydroprogesterone

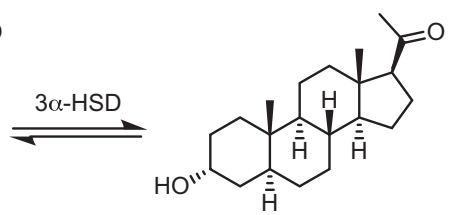

$3 \alpha, 5 \alpha-$ Tetrahydroprogesterone (Allopregnanolone)

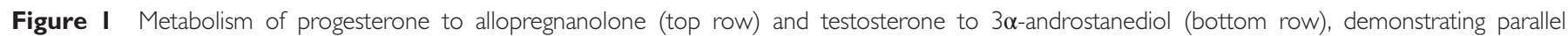
enzymatic pathways via $5 \alpha$-reductase and $3 \alpha$-hydroxysteroid dehydrogenase $(3 \alpha-H S D)$.

controls (Rasmusson et al, 2006; Romeo et al, 1998; Strohle et al, 1999; Uzunova et al, 1998). Girdler et al (2012) additionally noted decreased conversion of progesterone to allopregnanolone in women with a history of depression. Moreover, results from three small studies suggest that pharmacological antidepressant therapy with selective serotonin reuptake inhibitors (SSRIs) may increase allopregnanolone levels in CSF, serum, and postmortem brain, implying a possible mechanistic role in depression etiology and recovery (Agis-Balboa et al, 2014; Romeo et al, 1998; Strohle et al, 1999; Uzunova et al, 1998). One such study showed that SSRI treatment in depressed subjects led to an increase in CSF allopregnanolone levels and that these levels positively correlated with improvement in depressive symptoms in the absence of changes in the levels of precursors, such as progesterone (Uzunova et al, 1998). In another study, drug-naive outpatients with MDD demonstrated significant increases in serum allopregnanolone levels with SSRI treatment (Romeo et al, 1998). In a third study, serum allopregnanolone levels increased with 'clinically effective' antidepressant administration (Strohle et al, 1999). Studies have shown that the administration of $3 \alpha$-androstanediol reduces depressive behaviors in male and female mice (Frye and Walf, 2009). However, there are no data in humans on the testosterone metabolite $3 \alpha$-androstanediol in relation to mood disorders.

Anorexia nervosa (AN) and overweight/obesity (OW/OB) are both complicated by high rates of comorbid depression and anxiety. AN in women is complicated by depression and/or anxiety in $>50 \%$ of cases. Seven percent of obese adults have depression; $43 \%$ of adults with depression are obese (Bredella et al, 2014; Herzog et al, 1996). In women, increasing BMI has been associated with MDD and suicidal ideation, and the diagnosis of obesity has been shown to confer a $37 \%$ increased risk of an MDD diagnosis in women according to US National Survey data (Carpenter et al, 2000). Given the deficiency of gonadal hormonal substrates in women with hypothalamic amenorrhea, one might expect lower levels of neuroactive steroids in patients with AN. In contrast to this hypothesis, a few small studies have demonstrated higher allopregnanolone levels in women with AN vs healthy controls (Galderisi et al, 2003; Monteleone et al, 2001). Studies of neuroactive steroids in obesity are limited; two studies, one in women alone and one in both sexes combined, reported higher levels of allopregnanolone in obese than normal-weight individuals without consideration of comorbid psychiatric symptomatology (Menozzi et al, 2002; Monteleone et al, 2003). However, all these studies were limited by the use of immunoassays to measure allopregnanolone levels, which are particularly vulnerable to cross-reactivity with similarly structured steroid hormone metabolites (Cheney et al, 1995a; Siekmann, 1979). Additionally, while some studies exclude women on oral contraceptives (Monteleone et al, 2001; Monteleone et al, 2003) and control for the follicular phase of the menstrual cycle (Menozzi et al, 2002; Monteleone et al, 2001; Monteleone et al, 2003), others (Galderisi et al, 2003) do not specify these conditions, which are critical in the assessment of neuroactive steroids.

Given the sex steroid deficiency in AN, as well as the high rates of $\mathrm{AN}$-associated $\mathrm{MDD}$, we hypothesized that allopregnanolone and $3 \alpha$-androstanediol levels would be lower in women with AN than age-matched lean and overweight/ obese women; we also hypothesized that levels of allopregnanolone and $3 \alpha$-androstanediol would be inversely associated with severity of depression and anxiety across the three groups, independent of weight. We therefore measured serum levels of the neuroactive steroids allopregnanolone and $3 \alpha$-androstanediol using gas chromatography-mass spectrometry (GC-MS) performed after separation of the steroids of interest by high pressure liquid chromatography (HPLC) in women across the weight spectrum and investigated the relationship between these neuroactive steroids and affective symptom severity.

\section{MATERIALS AND METHODS}

\section{Subjects}

This study was approved by the Partners Healthcare Institutional Review Board and complied with Health Insurance Portability and Accountability Act guidelines. Written consent was obtained from all participants prior to study procedures. In order to facilitate investigation of these GABAergic neuroactive steroids across the weight spectrum, 36 women were 1:1 age matched across groups of 12 in each 
of the following BMI categories: (1) AN with BMI of $<18.5 \mathrm{~kg} / \mathrm{m}^{2}$, (2) healthy control (HC) with BMI $19-24 \mathrm{~kg} /$ $\mathrm{m}^{2}$, and (3) OW/OB with BMI $\geqslant 25 \mathrm{~kg} / \mathrm{m}^{2}$. Exclusion criteria for $\mathrm{HC}$ and $\mathrm{OW} / \mathrm{OB}$ subjects included patient-reported history of a depression diagnosis and/or antidepressant use. Additionally, none of the AN subjects were receiving antidepressant medications. The mean age among all groups was $26 \pm 6$ years. Formal evaluation for DSM-IV (American Psychiatric Association, 2000) disorders was performed after consent. In all cases, individuals meeting DSM-IV depression or anxiety disorders also met DSM-V criteria (American Psychiatric Association, 2013). Participants with anorexia nervosa were all amenorrheic. $\mathrm{HC}$ and $\mathrm{OW} / \mathrm{OB}$ women were all eumenorrheic and were studied in the follicular phase of the menstrual cycle. All subjects underwent a comprehensive medical history and physical exam as part of this protocol. Clinical characteristics of subsets of these subjects, but not this specific 1:1 age-matched group, have been reported as part of larger groups in parent studies, the following of which have reported HAM-D, HAM-A, or free testosterone levels (Bachmann et al, 2016; Brick et al, 2010; Eddy et al, 2015; Lawson et al, 2009a; Lawson et al, 2012; Lawson et al, 2010; Lawson et al, 2009b; Miller et al, 2007a; Miller et al, 2011; Miller et al, 2007b; Schorr et al, 2016). Neuroactive steroid levels have not been previously published in any parent study or subset analysis.

\section{Assessment of Mood Symptoms}

Hamilton Depression (HDRS; Hamilton, 1960) and Anxiety (HAM-A; Hamilton, 1959) Rating Scales were administered on the same day as the blood draw to measure symptom severity of depression and anxiety, respectively. Higher scores indicate greater severity of depression and anxiety symptoms. Clinically significant depression and anxiety in this study are defined as HDRS $>12$ and HAM-A $>14$, respectively.

\section{Hormone Analysis}

Samples were stored at $-80^{\circ} \mathrm{C}$ and run in one batch. Serum allopregnanolone, progesterone, and $5 \alpha$-androstane- $3 \alpha, 17 \beta$ diol (the $5 \alpha$ epimer of $3 \alpha$-androstenediol) were measured by
GC-MS after HPLC separation of the steroids of interest in the laboratory of investigator Graziano Pinna. The detection limit for allopregnanolone and the other neurosteroids determined with this method was approximately $5 \mathrm{fmol} / \mathrm{ml}$. The serum samples were extracted in ethylacetate and lyophilized; the neurosteroids of interest then were purified and separated with HPLC. Tritiated neurosteroids (American Radiolabeled Chemicals, St Louis, MO) were added to monitor retention time through the HPLC (Cheney et al, 1995b), while deuterated internal standards (CDN Isotopes, Pointe-Claire, QC; and Steraloids, Newport, RI) were added to allow quantification of the compound of interest. Because deuterated $3 \alpha$-androstanediol is not available, deuterated $3 \beta$ androstanediol was used as the internal standard for $3 \alpha$ androstanediol. Each steroid of interest was then derivatized for GC-MS (Pibiri et al, 2008; Pinna et al, 2000). Mass spectrometric analysis was conducted in the standard electron impact mode for all neuroactive steroid measurements. To calculate the quantity of the neurosteroid of interest in each fraction, the area under the peak of the neurosteroid in the sample was divided by the area under the peak of the deuterated internal standard.

Testosterone levels were measured at Esoterix Endocrinology (Calabasas Hills, CA) as follows: percentage of free testosterone was determined by equilibrium dialysis, with a minimum reportable free fraction of $0.1 \%$ and an intra-assay coefficient of variation of $8.8-9.4 \%$, and was multiplied by total testosterone levels as determined by tandem mass spectroscopy with a sensitivity of $3 \mathrm{ng} / \mathrm{dl}$ and intra-assay coefficient of variation of $0.72-17.3 \%$. The normal range for free testosterone levels in premenopausal women per Esoterix Endocrinology was 1.1-6.3 pg/ml.

\section{Statistical Analysis}

JMP Pro Statistical Database Software (version 11.0.0; SAS Institute, Cary, NC) was used for statistical analyses. Outliers [ $n=4$ for $3 \alpha$-androstanediol ( $2 \mathrm{AN}, 1 \mathrm{HC}$ and $1 \mathrm{OW} / \mathrm{OB})$ and $n=1$ for progesterone ( $1 \mathrm{OW} / \mathrm{OB}$, a different subject than the $3 \alpha$-androstanediol OW/OB outlier)] were excluded using JMP quantile analysis (below the first quartile minus 1.5 times the interquartile range or above the upper third quartile plus 1.5 times the interquartile range). Results are
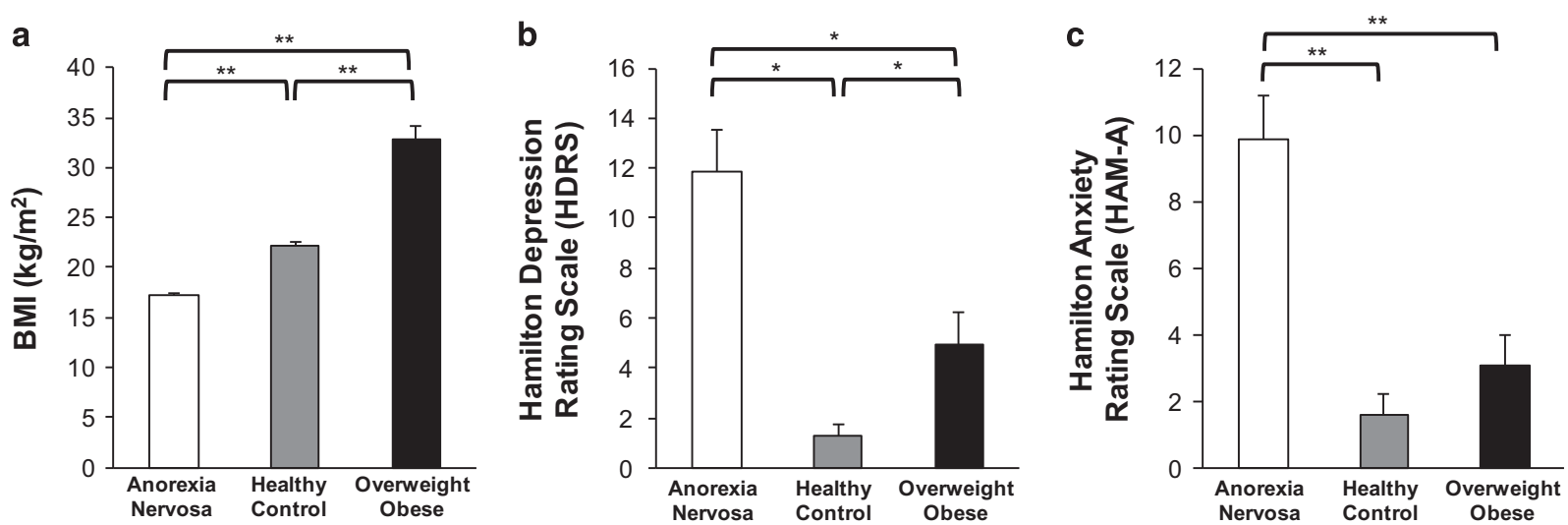

Figure 2 (a) Mean BMI $\left(\mathrm{kg} / \mathrm{m}^{2}\right)$ was significantly different between all three groups by design. (b) Mean depression symptom severity scores by HDRS were highest in AN, followed by OW/OB and HC. (c) Mean anxiety symptom severity scores by HAM-A were higher in AN but did not differ between OW/OB and $\mathrm{HC}$. $* *$ $p<0.000 \mathrm{I}$ and $* p<0.05$. Error bars indicate SEM. Abbreviations: AN, anorexia nervosa; HC, healthy control; OW/OB, overweight/obese; HDRS, Hamilton Depression Rating Scale; HAM-A, Hamilton Anxiety Rating Scale. 


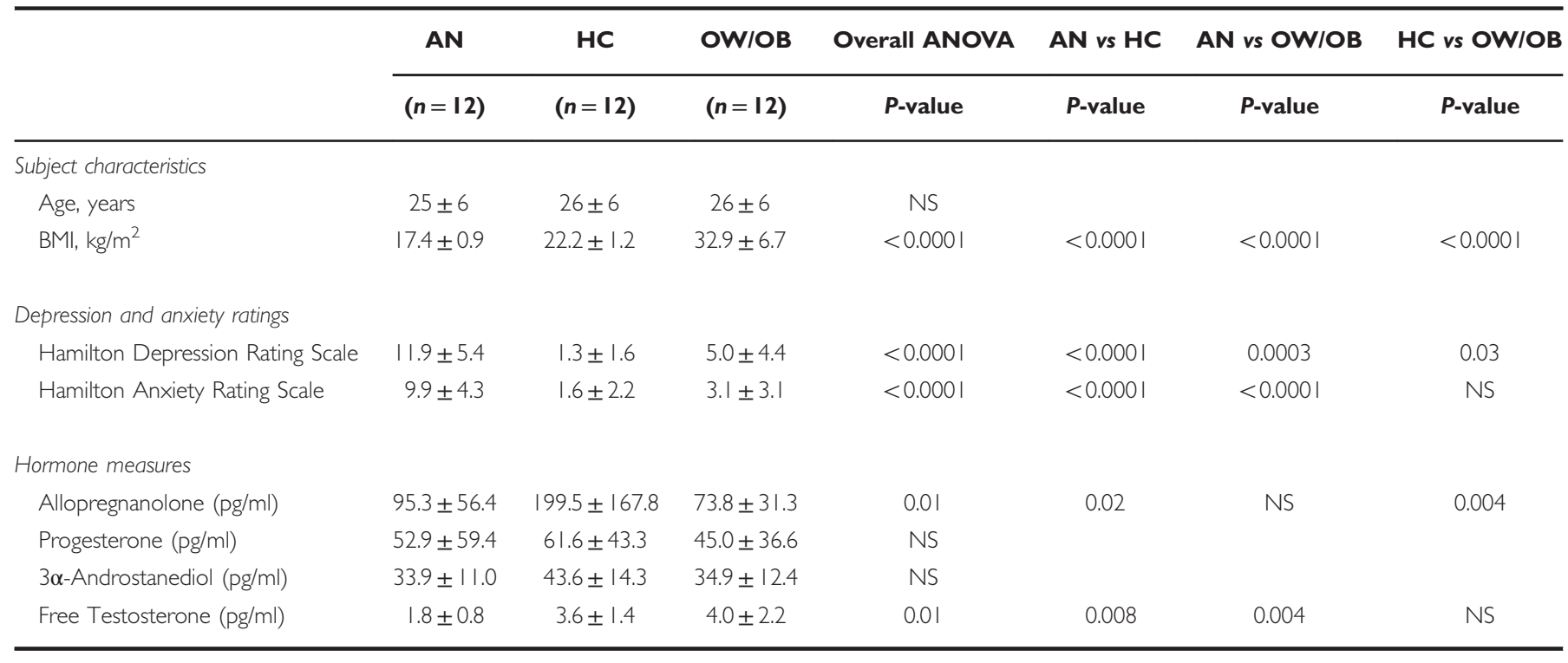

Abbreviations: AN, anorexia nervosa; ANOVA, analysis of variance; BMI, body mass index; HC, healthy controls; NS, not significant; OB, overweight/obese. Values reported as mean $\pm \mathrm{SD}$.

expressed as mean $\pm \mathrm{SD}$ unless otherwise noted. Variables were log-transformed. Means were compared across groups and were controlled for multiple comparisons with Fisher's least significance testing (Meier, 2006). Univariate regression analyses were performed with Pearson correlation coefficients reported. Multivariable analysis was performed using standard least squares or stepwise regression models.

\section{RESULTS}

\section{Clinical Characteristics}

There was no difference in mean age between the three groups. BMI differed among groups by design (AN $17.4 \pm 0.9, \mathrm{HC} 22.2 \pm 1.2$, and $\mathrm{OW} / \mathrm{OB} 32.9 \pm 6.7 \mathrm{~kg} / \mathrm{m}^{2}$, $p=0.0001$ ) (Table 1, Figure 2a). Smoking and alcohol use were known for $35 / 36$ subjects. There was only one current smoker in the AN group and none in the $\mathrm{HC}$ and $\mathrm{OW} / \mathrm{OB}$ groups. There were 19 subjects who used alcohol, including 5 of the AN group, 8 of the HC, and 6 of the OW/OB subjects, with a mean of 3.5 drinks per week in the subjects who did drink alcohol. The mean number of drinks per week did not differ between groups.

\section{Affective Symptomatology}

Mean HDRS scores were highest in women with AN, intermediate in $\mathrm{OW} / \mathrm{OB}$, and lowest in $\mathrm{HC}(\mathrm{AN} 11.9 \pm 5.4$ vs $\mathrm{OW} / \mathrm{OB} 5.0 \pm 4.4$ vs $\mathrm{HC} 1.3 \pm 1.6$, all pairwise $p<0.05$; Table 1, Figure 2b). Mean HAM-A scores were highest in women with $\mathrm{AN}$ and did not differ between women in the OW/OB and $\mathrm{HC}$ groups (AN $9.9 \pm 4.3$ vs OW/OB $3.1 \pm 3.1$ vs HC $1.6 \pm 2.2$, overall ANOVA $p<0.0001$; Table 1, Figure 2c). Overall, $36 \%$ of women with AN, no HCs, and $8 \%$ of the OW/OB women had clinically significant depression (HDRS $>12$ ), while $25 \%$ of women with $\mathrm{AN}$ and no $\mathrm{HCs}$ or OW/OB women had clinically significant anxiety (HAMA > 14).

\section{Allopregnanolone and Parent-Compound Progesterone}

Mean allopregnanolone levels were lower in women with AN and OW/OB women than HCs (AN 95.3 \pm 56.4 and OW/OB $73.8 \pm 31.3$ vs HCs $199.5 \pm 167.8 \mathrm{pg} / \mathrm{ml}, p=0.02$ and $p=0.004$, respectively; Table 1, Figure 3a). Mean serum progesterone levels did not differ between groups (Table 1, Figure 3b). Allopregnanolone levels were negatively associated with severity of depression (Figure 4a) and anxiety (Figure 4b), independent of BMI (HDRS whole model $R=-0.55, p=0.008$; partial correlation coefficient $R=$ $-0.46, p=0.008$; and HAM-A whole model $R=-0.56$, $p=0.04$; partial correlation coefficient $R=-0.37, p=0.04$ ). In multivariable analysis controlling for $\mathrm{BMI}, 15 \%$ of the variability in depression symptom severity and $11 \%$ of the variability in anxiety symptom severity were attributable to allopregnanolone levels. Within the AN group, strong, inverse associations with allopregnanolone were observed for depressive and anxiety symptoms, independent of BMI (HDRS whole model $R=-0.83, p=0.003$; partial correlation coefficient $R=-0.81, p=0.003$; and HAM-A whole model $R=-0.63, p=0.08$; partial correlation coefficient $R=-0.58$, $p=0.08$; Figure $4 c$ and $d$ ). There were no associations between allopregnanolone levels and depressive or anxiety symptoms within the $\mathrm{HC}$ or $\mathrm{OW} / \mathrm{OB}$ groups. There was no association between progesterone levels and depression or anxiety severity. There was a trend toward a positive association between progesterone and allopregnanolone levels $(R=0.33, p=0.09)$.

\section{3 $\alpha$-Androstanediol and Parent-Compound Testosterone}

Mean free testosterone levels were lower in the AN group than both the $\mathrm{HC}$ and $\mathrm{OW} / \mathrm{OB}$ groups ( $\mathrm{AN} 1.8 \pm 0.8 v s \mathrm{HC}$ $3.6 \pm 1.4$ and $\mathrm{OW} / \mathrm{OB} 4.0 \pm 2.2 \mathrm{pg} / \mathrm{ml}, \quad p=0.008$ and $p=0.004$, respectively; Table 1 , Figure $3 d$ ). In contrast, there was no difference in mean $3 \alpha$-androstanediol between any of the three groups (Table 1, Figure 3c). Free testosterone was negatively associated with HDRS scores $(R=-0.42, p=0.03)$ 

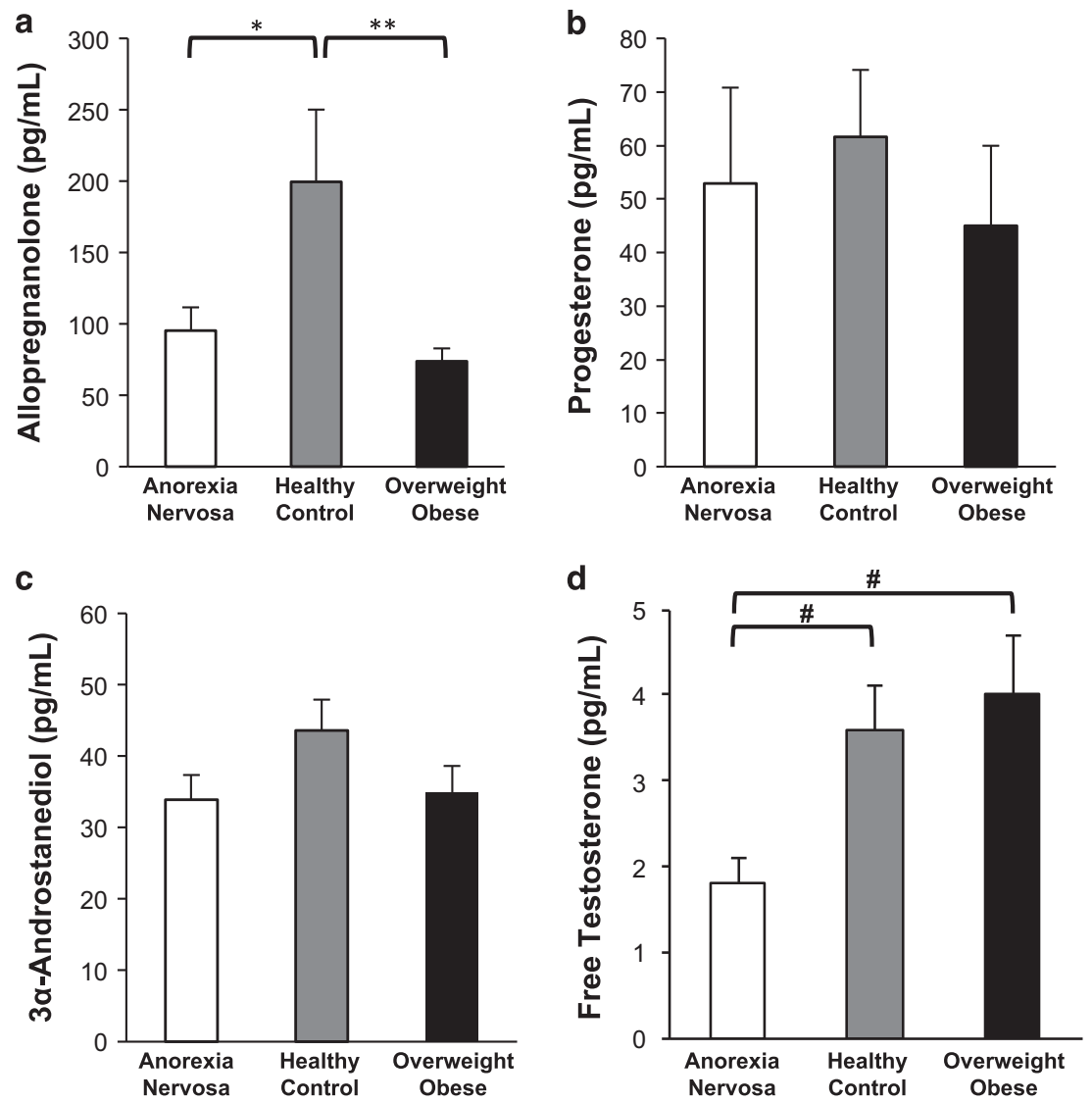

Figure 3 (a) Mean serum allopregnanolone levels were significantly lower in AN and OW/OB than in HC. (b) Mean serum levels of the parent compound progesterone did not differ across groups. (c) Mean serum levels of the neuroactive steroid $3 \alpha$-androstanediol did not differ between groups. (d) Mean serum levels of the parent compound testosterone was significantly higher in both OW/OB and $\mathrm{HC}$ vs subjects. ${ }^{*} p<0.05, * *{ }^{*} p<0.005,{ }^{\#} p<0.0$ I. Error bars indicate SEM. Abbreviations: AN, anorexia nervosa; $\mathrm{HC}$, healthy control; OW/OB, overweight/obese.

and HAM-A scores, $(R=-0.62, p=0.0006)$. The association of free testosterone with HDRS remained a trend and with HAM-A remained significant when controlling for BMI (HDRS whole model $R=-0.43, p=0.08$; partial correlation coefficient $R=-0.35, p=0.08$; and HAM-A whole model $R=-0.63, p=0.006$; partial correlation coefficient $R=$ $-0.53, p=0.006$ ). In a stepwise regression model, $18 \%$ of the variability in depression symptom severity and $39 \%$ of the variability in anxiety symptom severity was attributable to serum-free testosterone levels when controlling for BMI. Serum $3 \alpha$-androstanediol levels, however, were not significantly correlated with depression or anxiety symptom severity in any of the subgroups (AN, HC, or OW/OB) or the group as a whole. In addition, there was no association between free testosterone and $3 \alpha$-androstanediol levels.

\section{DISCUSSION}

The present study is the first to examine serum levels of the neuroactive steroids allopregnanolone and $3 \alpha$-androstanediol by GC-MS analysis in relation to affective symptoms in women with $\mathrm{AN}$ and $\mathrm{OW} / \mathrm{OB}$, both of which are frequently complicated by depression and/or anxiety. As hypothesized, our data show that mean serum allopregnanolone levels are lower in women with AN than in healthy controls. Unexpectedly, our data also demonstrate that mean serum allopregnanolone levels are lower in OW/OB women than in healthy women. Moreover, allopregnanolone levels were negatively associated with severity of depression and anxiety symptoms, independent of BMI. This cross-sectional study raises the question of whether a state of relative neuroactive steroid deficiency, possibly through downregulation of one of the two enzymes ( $5 \alpha$-reductase and/or $3 \alpha$-HSD), which convert the parent hormones to their neuroactive metabolites, may be a mechanism that contributes to the high rates of depression and anxiety at the extremes of the weight spectrum. Further studies are warranted to further explore this hypothesis.

Whether relative allopregnanolone deficiency is implicated in the pathophysiology of several psychiatric conditions, including depression (Romeo et al, 1998; Schule et al, 2007; Strohle et al, 1999; Uzunova et al, 1998), anxiety (Schule et al, 2014), and PTSD (Rasmusson et al, 2006), is an active area of investigation that has been limited by the lack of availability, high cost and time-consuming nature of GC-MS measurements. A small number of both cross-sectional and prospective treatment studies have examined allopregnanolone levels by GC-MS in the serum and CSF of subjects with MDD and PTSD, but no previous studies have explored the role of neuroactive steroids by GC-MS in women with AN or OW/OB. One small study of drug-naive men with MDD demonstrated significantly lower serum allopregnanolone levels compared with healthy controls (Romeo et al, 1998). 

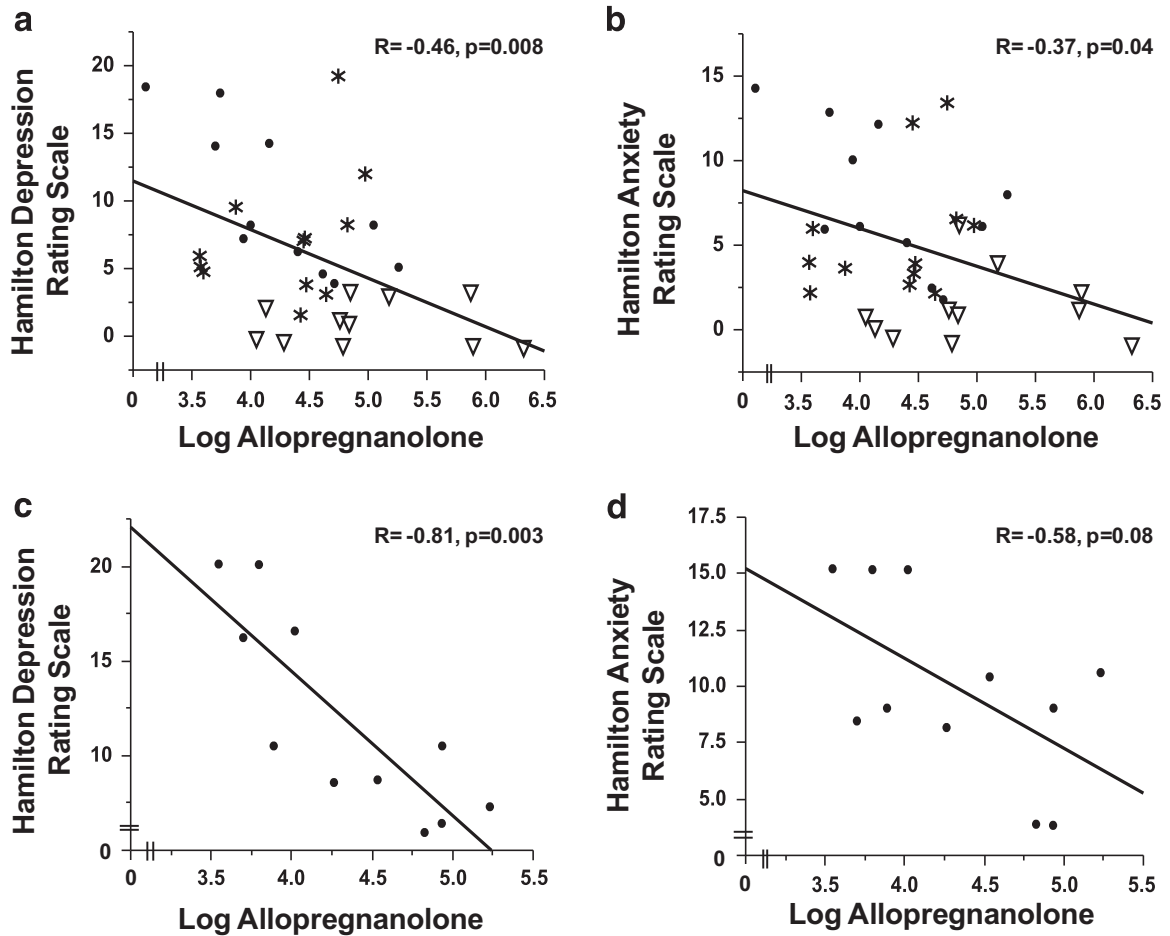

Figure 4 In the groups combined, serum allopregnanolone levels were significantly negatively associated with severity of depression (a) and anxiety (b) symptoms, independent of BMI. Serum allopregnanolone levels were also significantly negatively correlated with severity of depression (c) and anxiety (d, trend) symptoms independent of BMI in the anorexia nervosa group only. Figures adjusted for BMI (leverage plot shown) and partial correlation coefficients are reported on the figure. Markers: Closed circles indicate AN subjects, open triangle indicates $\mathrm{HC}$, and asterisk indicates OW/OB. Abbreviations: AN, anorexia nervosa; HC, healthy control; OW/OB, overweight/obese.

Studies have also shown a significant increase in serum allopregnanolone levels after successful treatment in small numbers of subjects with MDD, including drug-naive men administered fluoxetine (Romeo et al, 1998), previously treated outpatient subjects of both sexes given mirtazapine (Schule et al, 2007), and inpatients of both sexes treated with various regimens (including TCAs, SNRIs, and lithium) as determined by their individual clinical providers (Romeo et al, 1998). Moreover, CSF levels of allopregnanolone and its stereoisomer pregnanolone combined (termed 'ALLO') were negatively correlated with HDRS scores in men and women with MDD (Uzunova et al, 1998). The CSF ALLO levels in these subjects increased significantly after SSRI treatment, and the increase in ALLO was significantly correlated with improvement in depressive symptoms (Uzunova et al, 1998). In women with PTSD, CSF ALLO levels were reduced by $60 \%$ compared with healthy controls, with significant negative correlations between the ALLO/DHEA ratio and PTSD and negative mood symptoms (Rasmusson et al, 2006). Subjects with PTSD were found to have normal levels of the allopregnanolone precursor, $5 \alpha$-dihydroprogesterone, but a high $5 \alpha$-dihydroprogesterone/ ALLO ratio, suggesting that the decrease in ALLO was either due to a decrease in $3 \alpha$-HSD expression or activity or increased metabolism of ALLO. Thus, based on these data, there is great interest in the possible mechanistic contribution of allopregnanolone to mood symptoms.

However, two published studies demonstrated higher allopregnanolone levels in women with $\mathrm{AN}$ and bulimia nervosa compared with healthy controls (Galderisi et al, 2003; Monteleone et al, 2001), and authors postulated that hypothalamic-pituitary-adrenal axis activation in subjects with these disorders contributed to relatively higher allopregnanolone levels. However, all of these studies were limited by the use of radioimmunoassays, which lack specificity (Cheney et al, 1995a; Siekmann, 1979).

There are limited data with regard to neuroactive steroids across the weight spectrum. As hypothesized, our data show that mean serum allopregnanolone levels measured by GCMS were lower in women with AN than in healthy controls. Women with AN have low levels of sex steroids due to hypothalamic amenorrhea; thus one would potentially expect lower levels of neuroactive steroids due to less hormonal substrate, though progesterone levels may not differ from healthy, lean controls in the follicular phase. In contrast, OW/OB women can have normal to supraphysiological levels of hormonal substrate such as androgens and estrogens, and the only prior study that examined neuroactive steroids in obesity showed that otherwise healthy, obese adults had higher allopregnanolone than lean controls (Monteleone et al, 2003). Thus it was somewhat unexpected that serum allopregnanolone levels were lower in OW/OB women $v s$ healthy controls in this study. As noted earlier, methodological differences in measurement of allopregnanolone may have contributed to differences in the results between the current study and that by Monteleone et al (2003); in addition, it is possible that the OW/OB sample in the current study had a higher degree of affective symptoms.

Importantly, both the AN and $\mathrm{OW} / \mathrm{OB}$ groups had lower mean allopregnanolone serum levels and higher degrees of depressive symptoms than healthy, lean controls. Additionally, allopregnanolone levels were negatively associated with severity of depression and anxiety symptoms in women 
across the weight spectrum independent of BMI. Overall, these data suggest a possible mechanistic link between low levels of allopregnanolone and affective symptoms, independent of weight and differences in potential hormonal substrate between the two groups.

Progesterone, the parent compound to allopregnanolone, did not differ between groups across the weight spectrum in the current study, and again, there was no relationship between progesterone and affective symptoms, suggesting that the regulation of neuroactive steroid production downstream from progesterone-at either $5 \alpha$-reductase and/or $3 \alpha$ HSD - may be responsible for differences in mean allopregnanolone levels in these groups and have a key role in these conditions. This could be consistent with the work from Girdler et al (2012) showing decreased conversion of progesterone to allopregnanolone in women with a history of depression. Research in a frog model also shows that $\mathrm{GABA}_{\mathrm{A}}$ receptor activation can lead to a downregulation of neuroactive steroid production, suggesting a potential negative feedback loop; however, this has not been investigated in humans (Do-Rego et al, 2000). Thus, while neurosteroidogenic pathways are well defined, the overall regulation of neuroactive steroids is not yet fully understood.

In contrast to allopregnanolone, serum $3 \alpha$-androstanediol did not vary across the weight spectrum in this study, despite lower levels of free testosterone, the parent compound, in women with AN vs healthy and OW/OB women. Moreover, greater depression and anxiety symptom severity was not associated with serum $3 \alpha$-androstanediol, despite an inverse association of serum-free testosterone with both. The association between serum testosterone levels and anxiety symptom severity is consistent with prior work from our own group demonstrating an inverse association between testosterone levels and anxiety symptom severity in women with $\mathrm{AN}$ (Miller et al, 2007b). Furthermore, a significant acute improvement in depressive symptom severity was demonstrated in response to low-dose testosterone administration in women with AN in a pilot study (Miller et al, 2004). Moreover, hypogonadal men have a higher rate of comorbid depression, and testosterone administration in this population has been shown to improve mood, as well (Wang et al, 2004; Wang et al, 2000). Interestingly, the $5 \alpha$-reductase inhibitor finasteride has been associated with an increase in depression in some men, with studies showing a decrease in $5 \alpha$-reduced neuroactive steroids with treatment, as expected. Of note, however, these studies did not quantify the impact of finasteride on production of the $5 \alpha$-reduced metabolites of progesterone (Duskova et al, 2010). The $3 \alpha$-HSD gene possesses a steroid response element in its promoter, another possible site for interaction between testosterone or derivatives of testosterone such as estradiol and allopregnanolone synthesis in women (Mitev et al, 2003; Penning and Drury, 2007).

Limitations of this study include its small size and crosssectional design. In addition, we did not include normalweight women with a prior clinical diagnosis of depression and/or antidepressant use. We did measure hormones consistently in the follicular phase of the menstrual cycle, which is a strength of the study, as this limited the impact of large fluctuations of hormones such as estrogen and progesterone, the latter being the precursor for allopregnanolone. Additionally, testosterone is known to increase by approximately $20 \%$ at midcycle. Therefore, it is possible that we would have observed stronger or different associations in other phases of the menstrual cycle. It is also unclear whether peripheral levels of these neuroactive steroids accurately reflect central levels. With regard to testosterone specifically, the lack of a link between $3 \alpha$-androstanediol and affective symptom severity could indicate that $3 \alpha$-androstanediol is not the most relevant centrally active neurosteroid metabolite of testosterone. Given that the same enzymes convert testosterone and progesterone into $3 \alpha$-androstanediol and allopregnanolone, respectively, it remains surprising that only allopregnanolone levels were downregulated in AN and OW/OB women but not $3 \alpha$-androstanediol levels. Probably, enzyme affinity for the substrates or other biosynthetic mechanisms may be at play to dictate this apparent discrepancy. A limitation of this study is that we did not measure $5 \alpha$-dihydroprogesterone and $5 \alpha$-dihydrotestosterone; thus we cannot determine at which enzyme a block in the conversion of progesterone to allopregnanolone may have occurred. Finally, because our healthy controls and overweight/obese populations were selected for the absence of a depression diagnosis, our ability to detect an association between neuroactive steroids and affective symptoms may have been limited in these groups. Despite these limitations, this is the only study we are aware of that combines the use of careful follicular-phase sampling with GC/MS measurements.

In conclusion, women at both extremes of the weight spectrum have lower mean serum allopregnanolone levels than healthy lean women, despite a lack of difference in mean serum progesterone, the precursor of allopregnanolone. Moreover, greater depression and anxiety symptom severity is negatively associated with serum allopregnanolone, but not progesterone, independent of BMI. Lack of a strong association between allopregnanolone levels and the parent-compound progesterone suggests that there could be dysregulation of GABAergic neuroactive steroid synthesis or metabolism in depressed women with $\mathrm{AN}$ and $\mathrm{OW} / \mathrm{OB}$ women. More studies are needed to better understand the location and mechanisms of potential enzymatic dysregulation in neuroactive steroid pathways in women with both $\mathrm{AN}$ and $\mathrm{OW} / \mathrm{OB}$. Moreover, identification of centrally active neurosteroids that impact affective dysregulation could lead to a better understanding of the biological basis of depression and anxiety with the potential for the development of new, targeted therapies for these conditions.

\section{FUNDING AND DISCLOSURE}

Over the past 2 years, AMR received compensation as a member of the scientific advisory board for Resilience Therapeutics and as a consultant to Cohen Veterans Biosciences. KKM receives study drug from Marinus Pharmaceuticals. The other authors declare no conflict of interest.

\section{ACKNOWLEDGMENTS}

This work was supported by NIH Grants T32 DK007028, K24 HL092902, R01 MH083657, and R01 DK05262. This work was also conducted with support from Harvard Catalyst / The Harvard Clinical and Translational Science Center (National Center for Research Resources and the 
National Center for Advancing Translational Sciences, National Institutes of Health Award UL1 TR001102), National Center for Posttraumatic Stress Studies, Department of Veteran Affairs, and financial contributions from Harvard University and its affiliated academic healthcare centers. The content is solely the responsibility of the authors and does not necessarily represent the official views of Harvard Catalyst, Harvard University, and its affiliated academic healthcare centers, the National Institutes of Health or the Department of Veterans Affairs.

\section{REFERENCES}

Agis-Balboa RC, Guidotti A, Pinna G (2014). 5alpha-reductase type I expression is downregulated in the prefrontal cortex/Brodmann's area 9 (BA9) of depressed patients. Psychopharmacology (Berl) 231: 3569-3580.

American Psychiatric Association (2000). Diagnostic Statistical Manual of Mental Disorders. American Psychiatric Publishing: Arlington, VA, USA. 4th edn.

American Psychiatric Association (2013). Diagnostic and Statistical Manual of Mental Disorders. American Psychiatric Publishing: Arlington, VA, USA. 5th edn.

Bachmann KN, Schorr M, Bruno AG, Bredella MA, Lawson EA, Gill $\mathrm{CM}$ et al (2016). Vertebral volumetric bone density and strength are impaired in women with low-weight and atypical anorexia nervosa. J Clin Endocrinol Metab 102: 57-68.

Bredella MA, Fazeli PK, Daley SM, Miller KK, Rosen CJ, Klibanski A et al (2014). Marrow fat composition in anorexia nervosa. Bone 66: 199-204.

Brick DJ, Gerweck AV, Meenaghan E, Lawson EA, Misra M, Fazeli $P$ et al (2010). Determinants of IGF1 and GH across the weight spectrum: from anorexia nervosa to obesity. Eur J Endocrinol 163: 185-191.

Carpenter KM, Hasin DS, Allison DB, Faith MS (2000). Relationships between obesity and DSM-IV major depressive disorder, suicide ideation, and suicide attempts: results from a general population study. Am J Public Health 90: 251-257.

Cheney DL, Uzunov D, Costa E, Guidotti A (1995a). Gas chromatographic-mass fragmentographic quantitation of 3 alpha-hydroxy-5 alpha-pregnan-20-one (allopregnanolone) and its precursors in blood and brain of adrenalectomized and castrated rats. J Neurosci 15: 4641-4650.

Cheney DL, Uzunov D, Guidotti A (1995b). Pregnenolone sulfate antagonizes dizocilpine amnesia: role for allopregnanolone. Neuroreport 6: 1697-1700.

Do-Rego JL, Mensah-Nyagan GA, Beaujean D, Vaudry D, Sieghart $\mathrm{W}$, Luu-The $\mathrm{V}$ et al (2000). gamma-Aminobutyric acid, acting through gamma -aminobutyric acid type A receptors, inhibits the biosynthesis of neurosteroids in the frog hypothalamus. Proc Natl Acad Sci USA 97: 13925-13930.

Duskova M, Hill M, Starka L (2010). The influence of low dose finasteride, a type II 5alpha-reductase inhibitor, on circulating neuroactive steroids. Horm Mol Biol Clin Investig 1: 95-102.

Eddy KT, Lawson EA, Meade C, Meenaghan E, Horton SE, Misra M et al (2015). Appetite regulatory hormones in women with anorexia nervosa: binge-eating/purging versus restricting type. J Clin Psychiatry 76: 19-24.

Eser D, Romeo E, Baghai TC, di Michele F, Schule C, Pasini A et al (2006a). Neuroactive steroids as modulators of depression and anxiety. Neuroscience 138: 1041-1048.

Eser D, Schule C, Romeo E, Baghai TC, di Michele F, Pasini A et al (2006b). Neuropsychopharmacological properties of neuroactive steroids in depression and anxiety disorders. Psychopharmacology (Berl) 186: 373-387.
Frye CA, Walf AA (2009). Depression-like behavior of aged male and female mice is ameliorated with administration of testosterone or its metabolites. Physiol Behav 97: 266-269.

Galderisi S, Mucci A, Monteleone P, Sorrentino D, Piegari G, Maj $M$ (2003). Neurocognitive functioning in subjects with eating disorders: the influence of neuroactive steroids. Biol Psychiatry 53: 921-927.

Girdler SS, Lindgren M, Porcu P, Rubinow DR, Johnson JL, Morrow AL (2012). A history of depression in women is associated with an altered GABAergic neuroactive steroid profile. Psychoneuroendocrinology 37: 543-553.

Hamilton M (1959). The assessment of anxiety states by rating. $\mathrm{Br} \mathrm{J}$ Med Psychol 32: 50-55.

Hamilton M (1960). A rating scale for depression. J Neurol Neurosurg Psychiatry 23: 56-62.

Herzog DB, Nussbaum KM, Marmor AK (1996). Comorbidity and outcome in eating disorders. Psychiatr Clin North Am 19: 843-859.

King SR (2013). Neurosteroids and the Nervous System. Springer: New York, USA.

Lawson EA, Donoho D, Miller KK, Misra M, Meenaghan E, Lydecker J et al (2009a). Hypercortisolemia is associated with severity of bone loss and depression in hypothalamic amenorrhea and anorexia nervosa. J Clin Endocrinol Metab 94: 4710-4716.

Lawson EA, Miller KK, Blum JI, Meenaghan E, Misra M, Eddy KT et al (2012). Leptin levels are associated with decreased depressive symptoms in women across the weight spectrum, independent of body fat. Clin Endocrinol 76: 520-525.

Lawson EA, Miller KK, Bredella MA, Phan C, Misra M, Meenaghan E et al (2010). Hormone predictors of abnormal bone microarchitecture in women with anorexia nervosa. Bone 46: 458-463.

Lawson EA, Misra M, Meenaghan E, Rosenblum L, Donoho DA, Herzog D et al (2009b). Adrenal glucocorticoid and androgen precursor dissociation in anorexia nervosa. J Clin Endocrinol Metab 94: 1367-1371.

Majewska MD, Harrison NL, Schwartz RD, Barker JL, Paul SM (1986). Steroid hormone metabolites are barbiturate-like modulators of the GABA receptor. Science 232: 1004-1007.

Meier U (2006). A note on the power of Fisher's least significant difference procedure. Pharm Stat 5: 253-263.

Menozzi R, Florio P, Bondi M, Luisi S, Cobellis L, Genazzani AR et al (2002). Increased response of plasma allopregnanolone to corticotropin-releasing hormone in obese patients. Neuroendocrinology 75: 124-129.

Miller K, Grieco K, Klibanski A (2004). Testosterone administration in women with anorexia nervosa. The Journal of clinical endocrinology and metabolism 90: 1428-1433.

Miller KK, Lawson EA, Mathur V, Wexler TL, Meenaghan E, Misra $M$ et al (2007a). Androgens in women with anorexia nervosa and normal-weight women with hypothalamic amenorrhea. J Clin Endocrinol Metab 92: 1334-1339.

Miller KK, Meenaghan E, Lawson EA, Misra M, Gleysteen S, Schoenfeld D et al (2011). Effects of risedronate and low-dose transdermal testosterone on bone mineral density in women with anorexia nervosa: a randomized, placebo-controlled study. J Clin Endocrinol Metab 96: 2081-2088.

Miller KK, Wexler TL, Zha AM, Lawson EA, Meenaghan EM, Misra $M$ et al (2007b). Androgen deficiency: association with increased anxiety and depression symptom severity in anorexia nervosa. J Clin Psychiatry 68: 959-965.

Mitev YA, Darwish M, Wolf SS, Holsboer F, Almeida OF, Patchev VK (2003). Gender differences in the regulation of 3 alphahydroxysteroid dehydrogenase in rat brain and sensitivity to neurosteroid-mediated stress protection. Neuroscience 120: 541-549.

Monteleone P, Luisi M, Colurcio B, Casarosa E, Ioime R, Genazzani AR et al (2001). Plasma levels of neuroactive steroids are increased in untreated women with anorexia nervosa or bulimia nervosa. Psychosom Med 63: 62-68. 
Monteleone P, Luisi M, De Filippis G, Colurcio B, Genazzani AR, Maj M (2003). Circulating levels of neuroactive steroids in patients with binge eating disorder: a comparison with nonobese healthy controls and non-binge eating obese subjects. Int J Eat Disord 34: 432-440.

Morrow AL, Suzdak PD, Paul SM (1987). Steroid hormone metabolites potentiate GABA receptor-mediated chloride ion flux with nanomolar potency. Eur J Pharmacol 142: 483-485.

Penning TM, Drury JE (2007). Human aldo-keto reductases: function, gene regulation, and single nucleotide polymorphisms. Arch Biochem Biophys 464: 241-250.

Pibiri F, Nelson M, Guidotti A, Costa E, Pinna G (2008). Decreased corticolimbic allopregnanolone expression during social isolation enhances contextual fear: a model relevant for posttraumatic stress disorder. Proc Natl Acad Sci USA 105: 5567-5572.

Pinna G, Uzunova V, Matsumoto K, Puia G, Mienville JM, Costa E et al (2000). Brain allopregnanolone regulates the potency of the $\mathrm{GABA}(\mathrm{A})$ receptor agonist muscimol. Neuropharmacology 39: 440-448.

Rasmusson AM, Pinna G, Paliwal P, Weisman D, Gottschalk C, Charney D et al (2006). Decreased cerebrospinal fluid allopregnanolone levels in women with posttraumatic stress disorder. Biol Psychiatry 60: 704-713.

Reddy DS (2004). Testosterone modulation of seizure susceptibility is mediated by neurosteroids 3alpha-androstanediol and 17betaestradiol. Neuroscience 129: 195-207.

Reddy DS (2010). Neurosteroids: endogenous role in the human brain and therapeutic potentials. Prog Brain Res 186: 113-137.

Romeo E, Strohle A, Spalletta G, di Michele F, Hermann B, Holsboer $\mathrm{F}$ et al (1998). Effects of antidepressant treatment on neuroactive steroids in major depression. Am J Psychiatry 155: 910-913.

Rupprecht R, Holsboer F (1999a). Neuroactive steroids: mechanisms of action and neuropsychopharmacological perspectives. Trends Neurosci 22: 410-416.

Rupprecht R, Holsboer F (1999b). Neuropsychopharmacological properties of neuroactive steroids. Steroids 64: 83-91.
Schorr M, Thomas JJ, Eddy KT, Dichtel LE, Lawson EA, Meenaghan E et al (2016). Bone density, body composition, and psychopathology of anorexia nervosa spectrum disorders in DSM-IV vs DSM-5. Int J Eat Disord 50: 343-351.

Schule C, Baghai TC, di Michele F, Eser D, Pasini A, Schwarz M et al (2007). Effects of combination treatment with mood stabilizers and mirtazapine on plasma concentrations of neuroactive steroids in depressed patients. Psychoneuroendocrinology 32: 669-680.

Schule C, Nothdurfter C, Rupprecht R (2014). The role of allopregnanolone in depression and anxiety. Prog Neurobiol 113: 79-87.

Siekmann L (1979). Determination of steroid hormones by the use of isotope dilution-mass spectrometry: a definitive method in clinical chemistry. J Steroid Biochem 11: 117-123.

Sigel E, Steinmann ME (2012). Structure, function, and modulation of GABA(A) receptors. J Biol Chem 287: 40224-40231.

Strohle A, Romeo E, Hermann B, Pasini A, Spalletta G, di Michele F et al (1999). Concentrations of 3 alpha-reduced neuroactive steroids and their precursors in plasma of patients with major depression and after clinical recovery. Biol Psychiatry 45: 274-277.

Uzunova V, Sheline Y, Davis JM, Rasmusson A, Uzunov DP, Costa $\mathrm{E}$ et al (1998). Increase in the cerebrospinal fluid content of neurosteroids in patients with unipolar major depression who are receiving fluoxetine or fluvoxamine. Proc Natl Acad Sci USA 95: 3239-3244.

Wang C, Cunningham G, Dobs A, Iranmanesh A, Matsumoto A, Snyder P et al (2004). Long-term testosterone gel (Androgel) treatment maintains beneficial effects on sexual function on mood, lean and fat mass, and bone mineral density in hypogonadal men. J Clin Endocrinol Metab 89: 2085-2098.

Wang C, Swedloff R, Iranmanesh A, Dobs A, Snyder P, Cunningham $G$ et al (2000). Transdermal testosterone gel imprvoes sexual function, mood, muscle strength, and body composition parameters in hypogonadal men. Testostrerone Gel Study Group. J Clin Endocrinol Metab 85: 2839-2853. 